\title{
A Novel Approach to Pre-Impact Measurement from Impact Investing Using Random Forest and Deep Neural Networks
}

\author{
Emmanuel Kwesi Baah \\ Kwame Nkrumah University of \\ Science and Technology \\ Dept. of Computer Science \\ Kumasi - Ghana
}

\author{
James Ben Hayfron-Acquah \\ Kwame Nkrumah University of \\ Science and Technology \\ Dept. of Computer Science \\ Kumasi - Ghana
}

\author{
Dominic Asamoah \\ Kwame Nkrumah University of \\ Science and Technology \\ Dept. of Computer Science \\ Kumasi - Ghana
}

\begin{abstract}
Impacting investing is fast becoming an up-and-coming area in the finance industry. With the massive projection of investment that would go into this sector, there are present predicaments with the measurement of impact from impact investing, which casts doubts on the prospect of this concept. However, it is tagged as being characteristic of the future of investment. The challenge involves defining what to measure when to measure, and at what phase of investment. In this study, a combination of machine learning and deep learning models is used on the intended community to measure the preimpact factors suitable to generating confidence for the full granting of funds for impact investing. The first phase employed a survey of the impact community to gather features useful for the pre-impact assessment using redundant feature elimination with random forest. A deep neural network is then used to predict the various classes chosen for the classification problem. The results indicate that this new approach creates confidence in the next phase of impact measurement. Thus, the critical features for measuring the impact outcomes are not humanly generated or biased towards individuals but have a mathematical model that selects these features and the accuracy, precision, and recall for all three models are very significant. The deep learning and machine learning models had a unique advantage in resolving preimpact measurement from impact investing and proved promising for other investment phases with minimal human effort, cost-effectiveness and timeliness.
\end{abstract}

\section{General Terms}

Impact investing, Finance, Machine Learning

\section{Keywords}

Impact investing, random forest, deep learning, impact

\section{INTRODUCTION}

Over the past decade, there has been a resurgence in transforming many businesses and finance-related concepts. From the discussion of a group of industry pioneers typically leaders in development, philanthropy, and finance in 2007, the term impact investing was coined. They sought to evaluate not just with a financial lens what returns the capital invested could yield, however; the actual and potential performance. Twenty investors were invited by the Rockefeller Foundation and tasked to build a global industry and the need for such industries that strive to invest with a positive environmental and social impact [1]-[3]. Impact investing was targeted at generating financial returns while predominantly addressing environmental and social challenges shifting the focus away from only the financial objective of investment [3]. Since its inception, this concept has become promising in tackling pressing issues in society as it applies a holistic method to value creation that brings social impact and financial returns [1], [4].

Universally, there has not been any generally accepted definition for impact investing. However, several attempts have been made to address this. The Global Impact Investing Network (GIIN) definition of impact investing considers this an "investment made to generate positive, measurable social and environmental impact together with financial returns". The US has experienced a tremendous increase in impact investment Assets Under Management (AUM), doubling yearly and in early 2019 topped the US $\$ 502$ billion [5]. According to the report by [6], the Rockefeller Foundation, and J.P Morgan, impact investing is believed to become "one of the most powerful transformations in the industry of asset management", giving an estimate of close to $\$ 1$ trillion that would be channelled into this area by 2020 .

\subsection{Aim and Objectives}

This paper presents a new way of measuring impact from impact investing by a using machine learning approach to ascertain the pre-impact factors that investors and impact beneficiaries can consider before committing to an impact investing project. Random forest and deep neural networks are used to extract and predict the relevant sectors for impact investing.

\subsection{Background}

Although the promised impact investing has been shown to offer, one pressing challenge has been calculating the amount of capital that has been invested in global Social Impact Investing (SII) [7]. [8] identified that the investment mechanism and the diversity of capital absence or presence in the space of a unified exchange platform or market for social investment constitute the blockades and reasons for the challenge mentioned earlier.

[1] shared a similar trend with the complexity in measuring impact investing because there is a quest to standardize impact measurement in investment and other relevant approaches. What makes this even more challenging is that the objectives behind the measurement come with inevitable tensions; entrepreneurs and investors primarily deploy diversifying approaches in their measurement for diverse purposes. Similarly, both entrepreneurs and investors have divergent assessments of the opportunities and challenges of measuring impact investment - hence caution should be taken when measuring the social and environmental returns. 
[9] raise another issue regarding the assessment of impact investing. They argued that, though there is available helpful guidance for the measurement of impact investing according to the works done by [10] and [11], these are considered insufficient as the studies of [12], [13] projected. The challenge of enacting unified metric systems, assessment methods, and terminology is presently known as the embryonic phase and has been a critical challenge. This same study identified that no precise feedback mechanisms or incentives foster the quality of measuring impact investing. Thus, several approaches and conscientious means are used to measure financial returns and impact [9].

[14] shared that most of the approaches used by many organizations in measuring impact are still theoretical. From their analysis, limited resources discuss the precise methodologies and practices being used to measure impact by investors.

However, all these studies still do not vividly point out an approach categorized enough for investors to measure the impact before, during, and after the investment. Though mentions are made on the benchmarks for measurement [10], [11], the rigour and confidence with the measurement still require a more structured method to reap the full benefits of impact investing [12]-[14].

\section{LITERATURE REVIEW}

Though considered by many today as a new and emerging phenomenon in the finance industry, impact investing was traced to former times. In the $17^{\text {th }}$ century in England, the Quakers aimed at aligning the purchase decision and investment with their values. The 1800s also saw the Shaker's Congregations in Colonial America who targeted launching businesses that would yield social values to supply the religious communities of their time, which existed in many ways. Another trace is noted in the 1970s environmental movements, the 1980s anti-apartheid divestment camps, and advocate socially responsible investment and fair trade consumer goods, which have been a more recent movement all tracing to the pre-impact investing era that was conceived in 2007 [3]. Studies by [1] shared the history of the term 'impact investing' being rooted in the activities of the Rockefeller Foundation. In 2007, a group of leaders in development, philanthropy, and finance was convened in a meeting to reason out ways in which a worldwide industry could be built for investing for environmental and social impact. This meeting led to the coining of the term. Since the terms entered the public arena, their concrete definition remains a work in progress due to the many debates and diverse points of view by many investor groups and regions. However, the leading players in this field have made several attempts to offer a more rigour definition to impact investing.

Since the term 'impact investing' was born, there are attempts to consensus on a concrete definition [8]. However, one popular definition considered for this study on impact investing is Global Impact Investing Network (GIIN) as "investment made to generate positive, measurable social and environmental impact alongside a financial return". Impact investing may be considered 'accepting below-market returns' to accomplish some already set impact, while others consider the primary goal to achieve above-market returns with secondary attention to creating impact through the investment. The Calvert Foundation defined this as an investment that provides financial returns that are fixed but below market while economically inspiring the communities around [15].

There are several approaches to measuring impact from impact investing; most of these methodologies are viewed as technical and have not reached the point of addressing what to measure, when to measure, and by whom [16].

Although the projected benefit of impact investment is to generate a positive social impact for the community involved along with financial returns, investors struggle to measure this 'social impact' as a baseline for directing and assessing investment decisions and proving the investment's success. Unlike financial impact, where standardized methodologies are available for measurement, such as return on investment, the same cannot be said of social impact [17], [18].

Measuring impact varies across different spectrums among intended beneficiaries, investees, impact investors. In contrast, some may consider meeting the Sustainable Development Goals (SDGs) as a reference point that constitutes a "positive impact and offer a unifying ground across the different institution [18], [19]. These goals are not specific measurement strategies because they are vast [19].

[15] shared some critical challenges in measuring impact, some of which are discussed here. Investors are challenged with measuring the impacts of each investment due to the uniqueness that comes with them. Some investment creates an 'impact' in some cases that would not fit the generalized standard measurement frameworks like the GIIRS and IRIS. Apart from knowing how to measure the impact, it is also a challenge to the investor to utilize the measured impact. Some investors tie the additional return of capital to having achieved predefined impact outcomes, while others may decide to waiver interest rates (in the case of debt) or reward management with performance bonuses (in equity investments).

Lastly, the fear of 'greenwashing' of the impact sector and watering impact goals and noble community mission must be carefully considered. At the same time, an accurate measurement is also of great concern [15].

One predominant challenge to measuring impact, should there be a consensus among investors, would be the demand of efforts, money, and time involved for those undertaking impact activities and monitoring. Hence, there are limited metrics or indicators for measuring social impacts as they resort to the 'economic value created' or the 'lives touched' [20].

Recent advancements and contributions by deep learning in many fields of study, particularly in speech recognition and image processing, have aroused interest in this area. It also looks promising in other domains like big data analytics [21], [22]. These transformations are evident in daily lives, with tremendous impacts on speech recognition, predictive forecasting, self-driving cars, precision medicine, and cancer diagnosis [23]. Deep learning is mostly applied to visual and audio or audio-visual data and numerical and text data for solving semi-supervised, unsupervised, regression, and recognition problems due to its capability to learn hierarchical features from the data [24]-[26].

Though deep learning can potentially learn from labelled data provided a quantum amount of data is available, given a large amount of unsupervised/unlabelled data, it is mainly striking to note that it can extract patterns and meaningful representation from big data [27]-[29]. In this study, considering the unstructured nature of data received on impact investing, deep learning is the best methodology to draw powerful meaning from these data. 
Unlike the other methodologies considered previously, one study, the Social Impact Measurement Model (SIMM), a UK Private Company called Deloitte Insights, is uniquely developed using machine learning to assess the impact created from corporate spending. It is used to accurately predict the outcome of significant investments and the happening in the absence of the investment. The model analyses 142 social measures in the US ranging from reading proficiency and child poverty, population, migration by estimating the social impact of the investments done at the county level over four years. The SIMM provides a better understanding of what investment impacts more or lesser than others. It is vital in decision-making by community leaders, investors, policymakers, and other stakeholders [30].

This model works by combining Deloitte's proprietary data on corporate investments from the counties against data available in the public domain on the 142 socioeconomic features and begins with the county-level socioeconomic indicators. Thus, it does not separate an investment as the leading cause of a transformation experienced in a social measure. Counties with or without investments are compared by finding matched pairs within the timeframe specified, choosing the paired counties as those of like similarities as possible at the end of the period. Owing to the similarity in the paired counties', any social measures changes are regarded as an economic investment. The baseline for this philosophy is that though many reasons will account for social measures, these factors will behave equally in both counties apart from the fact that they are influenced by investment [30].

[30] again share that this approach to impact measurement only deals with estimates that augment the established methods. Some have been well-elaborated previously that consider information gathering, conducting analyses, and generating insights helpful in allocating capital and planning purposes. The SIMM is a statistically rigorous and quantitative way of showing the connection between financial investment and social outcomes, which happens to be the first of its kind in the methodologies used in impact measurement.

[11] first published their work on various organizations' various impact measurement methods. This study considered and reviewed over 20 catalogues of approaches that have been used in impact measurement. A detailed explanation of the methodology, the scope of analysis used, its application to date, and its feasibility to measure impact. Lastly, it considers credibility and verifies the processes involved in impact measurement. [9] followed with a study to prove that the measurement of impact has not found a systematic, participative, and pragmatic way forward, opening up critical gaps that need to be addressed to advance impact impact impact investing. With this, [14] reviews some critical gaps in the principal methodologies that the study considered fundamental approaches and designed a framework that deepens the understanding of the methodologies and practices used by established impact investors. These studies acted as the baseline for the study as this study seeks to employ a deep learning approach to measuring impact investing. However, [31] studied the measurement process of impact investing, considering Europe as the focus. The author focused on addressing the state of impact investing in Europe through a survey. Analysis of the results indicated that Europe's approach to measuring impact is underdeveloped. Lastly, Deloitte Insight reported another approach that employs machine learning to measure impact [30]. Hence, this study considered the insight drawn from the challenges encountered so far concerning the impact and how they can be addressed using deep learning since it has some advantages over machine learning.

The study by [14] evaluated the pitfalls of the methodologies considered for measuring impact, namely the expected returns, the theory of change and logic model, experimental and quasi-experimental methodology, and the proposed mission alignment methods integrated approach. However, this integration still fails to consider the time, efforts (human resource), and cost investors and investees use in measuring impact.

From the review, it has been revealed that deep learning has some key features that can learn of unstructured data generated through the process of investment; this confidence was boosted by the work done by [30] with the use of machine learning in measuring the social impact in impact investing. Thus, applying appropriate machine learning and deep learning models would draw proper insight into data generated to measure impact.

\section{METHODOLOGY}

To minimize and design a more efficient, credible, and unbiased methodology for evaluating impact from impact investing, deploying combined algorithms in machine learning and deep learning powerful at data analysis from big data would be significant as this sector harnesses diverse kinds of data. Thus, the diversity of the kinds of data involved in measuring impact demands a new approach that would quantify the impact generated, building much confidence for investors. Hence, using a combination of these machine learning and a deep neural network deployed at each phase to address the data used. The Python programming language was used for all the experiments carried out in this study. Figure 1 shows the conceptual framework for this study.

In this paper, the researcher employed machine learning and a deep neural network to achieve the objectives of this study. First, the relevant data is gathered through a survey from the three key stakeholders, including the impact investor, the impact creator, and the impact beneficiaries. Then the Redundancy Feature Elimination with Random Forest (RFRFE) algorithm is used to harness the relevant sets of features, and lastly, a deep neural network has deployed the prediction of the critical classes.

\subsection{Data Acquisition}

Since getting real-time data from the major stakeholders on impact investing was challenging, as shared in the review regarding the centre at GIMPA. A new structure for addressing the concerns with pre-impact measurement was designed. The study area chosen for evaluating and determining impact was Kwame Nkrumah University of Science and Technology (KNUST).

According to [1], the three principal deficiencies with measuring the social and environmental returns on investment were what to measure, who does the measurement and at what stage and how it is measured. The premises for enacting the questionnaires for getting the hypothetical data for the preimpact investment prediction for the social and environmental returns were based on the study.

The questionnaire was sectioned into three main blocks with critical questions which are targeted at assessing what can be measured as an impact in the chosen area, the perspective of the stakeholders or beneficiaries for the investment, and the likelihood of predicting the relevant features to look out for in awarding funds for impact investment. 


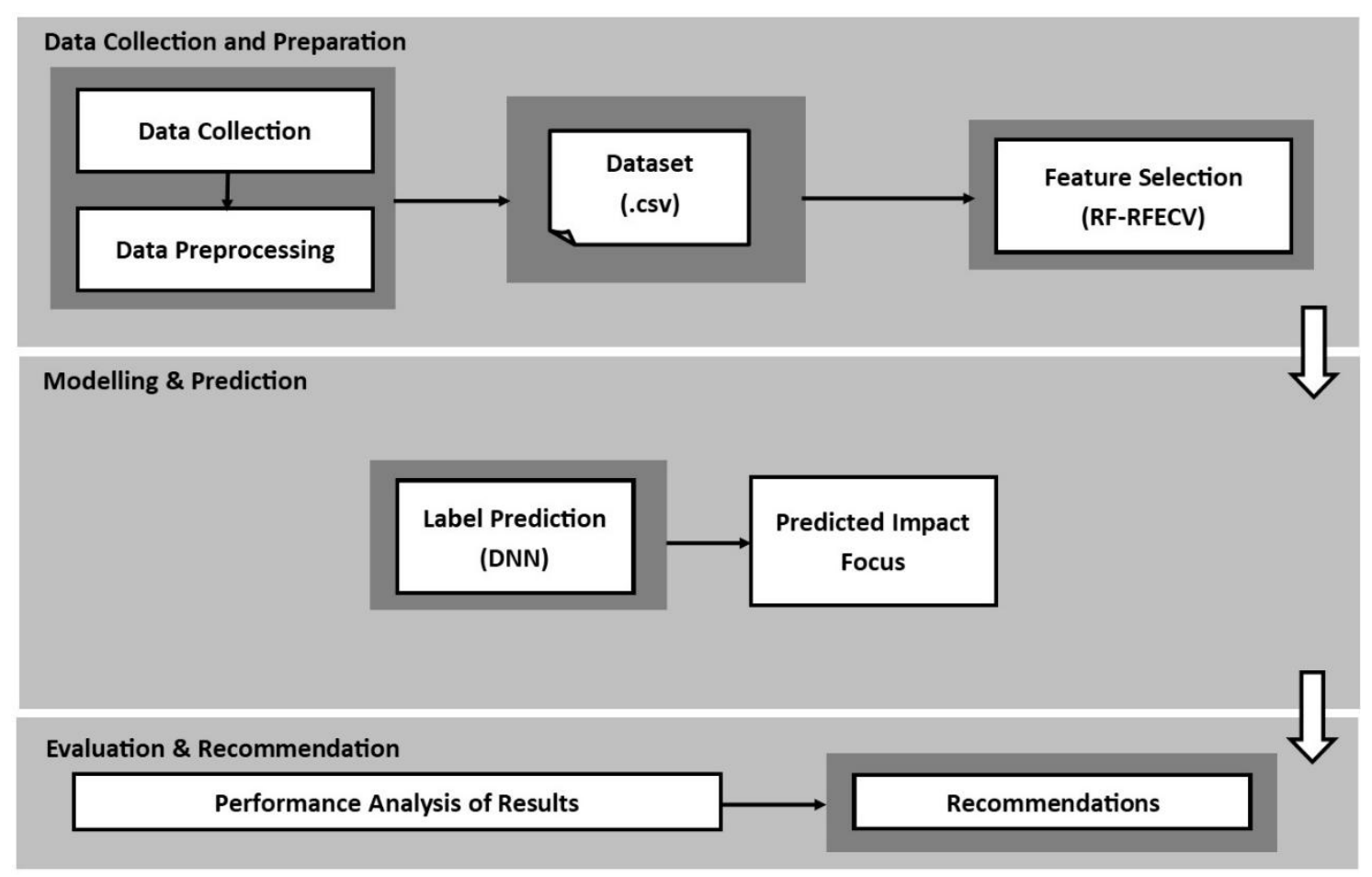

Fig 1: Conceptual Framework

\subsubsection{Description of Dataset}

As the study by [30], the data gathered from the sampled population of students and staff from KNUST was to harness the relevant social measures pivotal in informing stakeholders about the likely profitability of a business model determined to generate some social and environmental returns on investment. These social measures were taken from the Sustainable Development Goals (SDGs) and Millennium Development Goals (MDGs) obtained from the official website and documents obtained from the Research Centre at GIMPA. The reason for this was to allow for the chosen environment around the university to see the potential benefits from the investment in its surrounding neighbours. Next, the present predominant challenges facing the university with a high potential of attracting funding from investors were also considered in the questionnaires. Significant social measures considered for analysis were education, employment, income, agriculture, forestry, energy, Technology, and living standards. A snapshot of these features is represented in Table 1 .

\subsubsection{Data Pre-processing}

Before the dataset could be trained and used for prediction, there were some data pre-processing. About five hundred and sixty (560) records were obtained from the survey as hypothetical datasets to evaluate the three perspectives of measuring impact from impact investment; impact investor, impact creator, and impact beneficiaries. Most of the questions given were closed-ended to get a restricted audience and help assign numerical values to these features. After the rework, only five (five) were deleted from the sample because they were the responses did not conform to the study's objectives, and all the responses are given were converted to numerical values using Label Encoders to prepare for the machine learning algorithms to be used on the dataset for feature selection, and classification.

Thus, this process was vital to ensure that, given an area (community, country, city) chosen for impact investing, what relevant indicators would trigger the desired impact. Hence, these machine learning algorithms used to select relevant features and predictions would become the key indicator for assessment during the pre-impact measurement.

\subsection{Feature Selection}

Feature selection before training the model is vital in any artificial intelligence research. It aims to choose a subset of relevant features for the prediction given the original features by eliminating noisy, redundant, irrelevant features. Thus, there is usually an improvement in the performance of the analysis during the learning phase. Some key advantages to this technique are better model interpretability, lower computational cost, and higher learning accuracy [32].

Feature selection was necessary due to the text classification after predicting the key features relevant to the classification problem. It helps trim the number of features in the dataset and enhance the training time and accuracy of the model.

The Random Forest - Redundancy Feature Elimination CrossValidation (RF-RFECV) was used for selecting relevant features in the dataset. In all, fifteen (15) features were gathered.

\subsubsection{Random Forest-Recursive Feature Elimination (RF-RFE)}

Random Forest(RF), proposed by [33], is one of the most used classifiers comprising many decision trees and is also considered a meta-learning algorithm. When this classifier is used for training, it employs the bootstrap resampling methods to extract a relevant subset of features. Finally, the results are determined by the score obtained by every decision tree. The classification error lies in each tree's power to classify and the relationship between the trees [34]. Random forest is a powerful model for classification, which has seen many applications in diverse fields [35], [36]. Compared to other machine learning algorithms combined with RFE, it has proven better, especially with Support Vector Machine (SVM). 
However, the overall process is similar [37]. This study minimized the feature space dimension using the RF algorithm, joining it with the RF-RFE. It is done to reduce redundancy in the data and achieve more significant feature subsets.

\section{The RF-RFE algorithm in summary is:}

i. Train the features using RF with the current dataset.

ii. For all features, acquire the feature importance of every feature.

iii. Remove all features scoring the minor importance.

The RF-RFE repeatedly implements this process to obtain optimal feature subsets, which is crucial with cross-validation. For evaluation purposes, cross-validation was done to avoid overfitting the dataset. Figure 2 shows the score of the selected features and the number of features selected.

\subsection{Deep Neural Network for Classification}

Deep neural networks have a significant advantage over machine learning. For the classification model whose output would be used for the text classification in the next phase, deep neural networks were chosen for this approach. The deep neural network used for the classification problem used three (3) hidden units (neurons) of 20, 30, 50. The classification problem number was five (Technology, Energy, Agriculture, Health, and Education). To further check against losses and provide a more accurate result, the Adam optimizer was used for tunning the results. Lastly, the ReLU function, one of the most used transfer functions, utilized the essential information and suppressed irrelevant data entries.

The following steps expressed how the network in each layer is propagated [38];

i. Calculate the weighted sum of each neuron, thus multiplying the respective weight of the connection with that neuron with the output value of each neuron in the proceeding network layer.

ii. Apply a transfer function $f(s)$ to the weighted sum to get the value of the neuron's output.

iii. Express the output value $y$ as a function of the network weights and input values.

\subsection{Performance Metrics}

For evaluation purposes of this study, significant performance metrics were chosen for the two phases. For the first phase, which predicts the necessary features to focus on for the text classification, the accuracy, precision, and recall were the main focus on how the model formed [39].

\section{DISCUSSIONS}

From the list of features discussed above, trying other classifier combinations with the RFE such as the support vector machine, decision trees, and logistic regression, it was observed that the RF-RFE performed better with the score used for the selection of the relevant features as well as selected the most significant features from the list of options where were gathered from the sources as the MDGs and SDGs. Hence, RF-RFE was ideal in selecting relevant features useful for the classification problem. The fifteen (15) features from the dataset obtained, only selected twelve (12) of them were considered optimal by the RF-RFE Figure 2. All these features selected were in the first rank. The accuracy score of the RF-RFE is $99 \%$ indicating the highest performance in the experiments. It took the model approximately 103 seconds to select these features. Thus, these three features which were eliminated did not influence the prediction by the deep neural network. When newly selected features were used for the classification with Deep Neural Networks (DNN), 30\% of the dataset was used for the testing, while $70 \%$ were used for the training. As shared earlier, the model was tested to check its performance. The results of the various labels used were obtained using the Confusion Matrix. It can be observed that all the critical features from the three impact stakeholders were chosen, signifying that the model was able now to harness the most relevant features without any human reasoning. These selected were used to predicting which focus the impact investment in the chosen area, KNUST, with the five classes being Technology, Energy, Agriculture, Health, and Education. Table 2 shows the performance of the DNN model on the various labels. The overall accuracy score was approximately $92 \%$ for all the labels. It was observed that the AUC (Area Under Curve) interpretation for all the labels was excellent. They all score at more than $90 \%$. The precision and recall recorded for all the labels for Technology and Health being below $90 \%$ shows there needs to be much work to predict these classes. Thus, the number of false-positive recorded were high. The Education and Energy sector had a perfect score for all the records. With the performance list for the accuracies scores relatively high for all the labels, the DNN was ideal for the classification problem. Thus, these results give impact investors the confidence of the area to invest in KNUST. It would have to do with accepting business models or projects with more or combinations of these areas, as these have been well refined and extracted using an unbiased approach. This result indicates that the model performed exceptionally well for almost all the labels. Thus, considering the Confusion Matrix given in Figure 3, it observed that most of the features were classified under Technology (106 records) over the 167 records used for the testing. Thus, an investment focus for KNUST would possibly be looking at projects that seek to apply innovative Technology and generate impact for the community. Next, the Agriculture sector (30 records) also had many features classified. Though Education and Energy had a $100 \%$ score for all the metrics, it was key to note that the number of records selected for these was low Figure 4 shows the overall performance of the DNN model. It indicates and affirms that investors should focus on models that align with the most predicted label (thus Technology and Agriculture) and correctly projects which sector investors can be on the lookout for in the next phase of the prediction using natural language processing to rate impact creators' portfolios in other to measure the social and environmental returns on their investments truly. Again, the results from the study indicate that an acceptable performance of the DNN model though, it records some false positives for the two highly predicted labels (Technology and Agriculture).

\section{CONCLUSION}

In this study, a new approach to pre-impact measurement is designed and tested, which to the authors' knowledge, is the first approach combining machine learning and deep learning aside from the study by [30]. Using random forest with redundancy feature elimination brought out the relevant features necessary for predicting the labels. Thus, the accuracy, precision and recall achieved were enhanced. The deep neural network algorithm also recorded a high accuracy and minimal false-negative, boosting the prediction's confidence. For future studies, the studies use the most predicted label and training a deep learning model that would be used to classify business models or impact-intended 
documents to predict their impact on the chosen community; hence impact investors can hasten the process of awarding impact funds by developing an automated machine learning application that selects the features and makes a prediction and displays how one impact creators model is more impactful than the other.

Table 1. Features Selected for the Prediction

\begin{tabular}{|c|c|}
\hline Features & Labels \\
\hline support_for_impact_investing_in_ghana & $\begin{array}{l}\text { unemployment, health_problems, electricity_issues, sanitation_challenges, } \\
\text { teengage_pregnancy, poverty_alleviation }\end{array}$ \\
\hline previous_impact_contributors & $\begin{array}{c}\text { agriculture, sanitation, poverty_alleviation, ground_breaking_research, } \\
\text { graduate_employement, energy_sector }\end{array}$ \\
\hline addressable_impact_issues & $\begin{array}{l}\text { energy_crisis, preserving_forest_reserves, climate_change_projects, } \\
\text { creating_jobs_for_youth, improving_standards_of_living }\end{array}$ \\
\hline impact_creator_attributes & social_impact_recorded, credibility, completed_projects, ranking_or_rating \\
\hline critical_issues_for_awarding_funds & $\begin{array}{c}\text { energy_crisis, alumni_graduate_unemployment, } \\
\text { psychological_challenges_with_students }\end{array}$ \\
\hline most_significant_area_of_impact_creator & $\begin{array}{c}\text { graduate_unemployment, power_challenges, psychological_challenges, } \\
\text { support_for_innovative_research, support_for_brilliant_students }\end{array}$ \\
\hline impact_recognition_channels & official_report, observation, social_media, news_report \\
\hline perceivable_years_for_impact_measuring & one, two, three, four, five, ten, above_ten \\
\hline preffered_feature_for_impact_investing & $\begin{array}{c}\text { achieve_gender_equality, end_poverty, climate_change, } \\
\text { combat_endemic_diseases, health_life_promote_well_being_for_all, } \\
\text { promote_sustainable_economic_growth }\end{array}$ \\
\hline critical_impact_factors_on_environment & $\begin{array}{l}\text { addressing_youth_unemployment, handling_road_traffic_issues, } \\
\text { health_problem, ict_infrastructure, poverty, teenage_pregnancy }\end{array}$ \\
\hline pressing_concerns_in_impact_area & $\begin{array}{l}\text { agriculture, accommodation, health problem, quality_eduction, research } \\
\text { support, support_for_needy_students, technological_innovation, } \\
\text { transportation, }\end{array}$ \\
\hline chosen_impact_sector & agriculture, education, energy, health, technology, \\
\hline
\end{tabular}

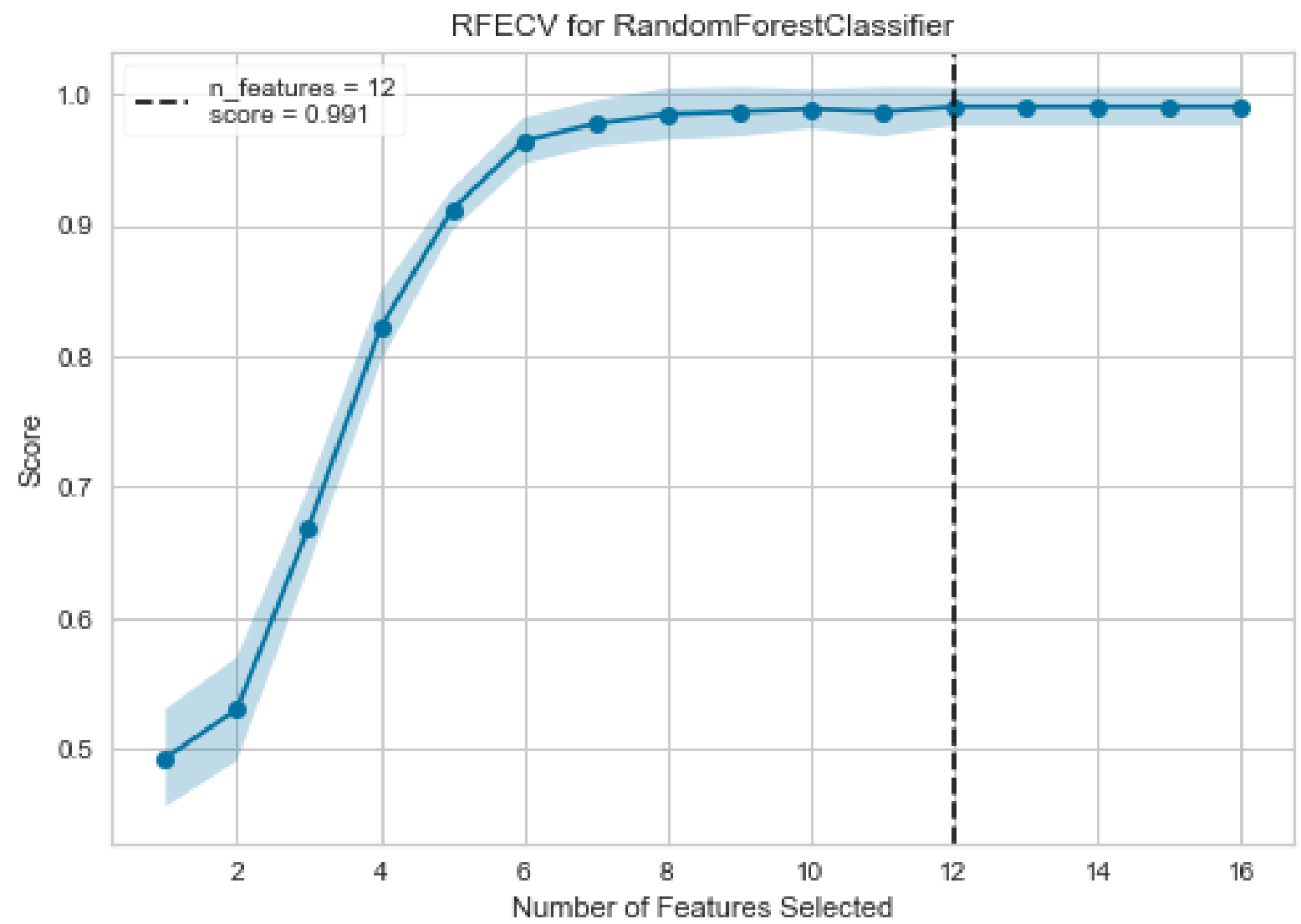

Fig 2: Graphical View of the Twelve Selected Features 


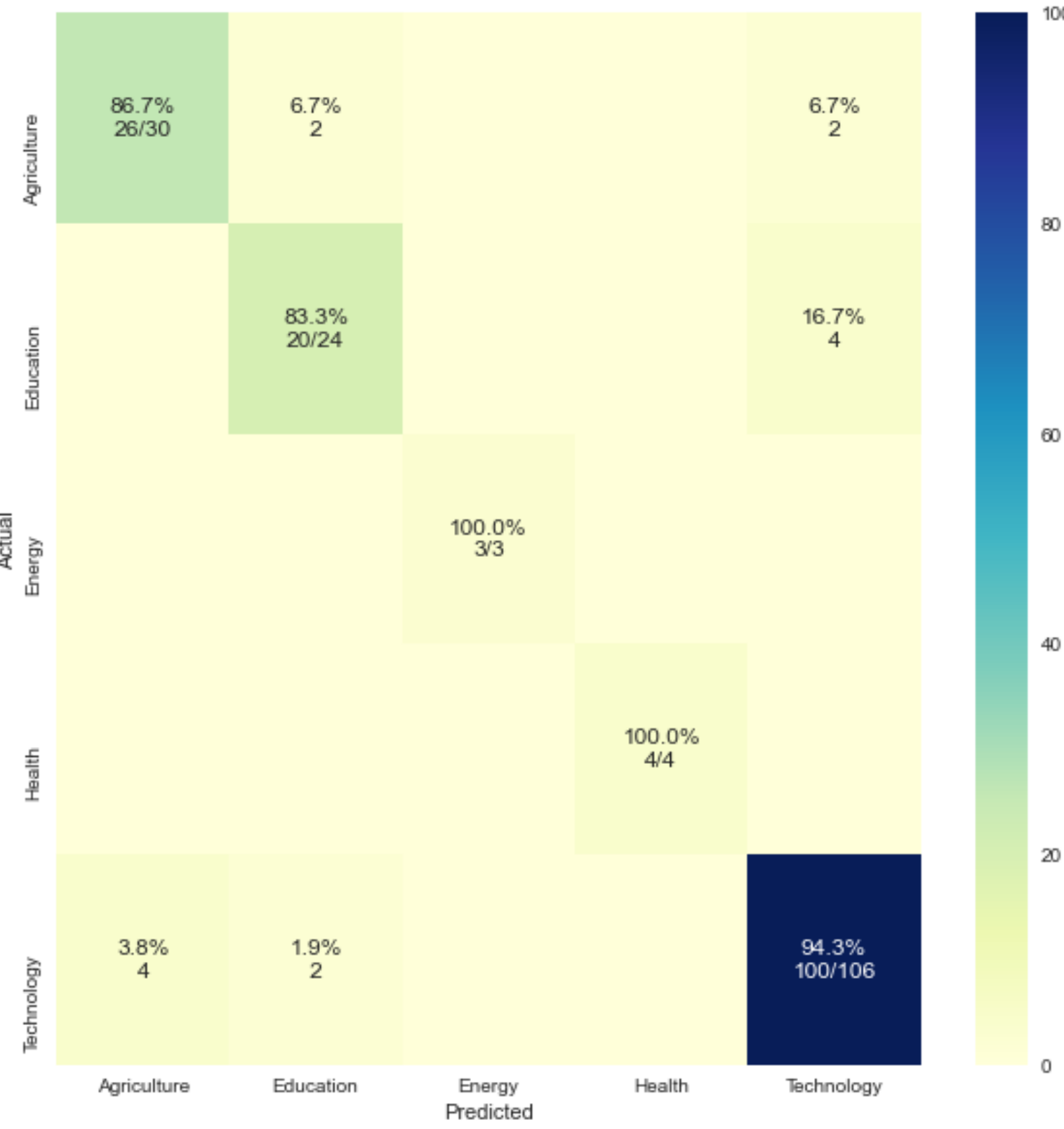

Fig 3: Confusion Matrix of the Five-Predicted Classes

Table 2. Accuracy, F1-score, Precision, Recall and AUC of the Predicted Label

\begin{tabular}{|c|c|c|c|c|c|}
\hline & Technology & Health & Energy & Education & Agriculture \\
\hline Accuracy & 0.952 & 0.952 & 1.000 & 1.000 & 0.928 \\
\hline F1-score & 0.867 & 0.833 & 1.000 & 1.000 & 0.943 \\
\hline Precision & 0.867 & 0.833 & 1.000 & 1.000 & 0.943 \\
\hline Recall & 0.867 & 0.833 & 1.000 & 1.000 & 0.943 \\
\hline AUC & 0.919 & 0.903 & 1.000 & 1.000 & 0.923 \\
\hline
\end{tabular}




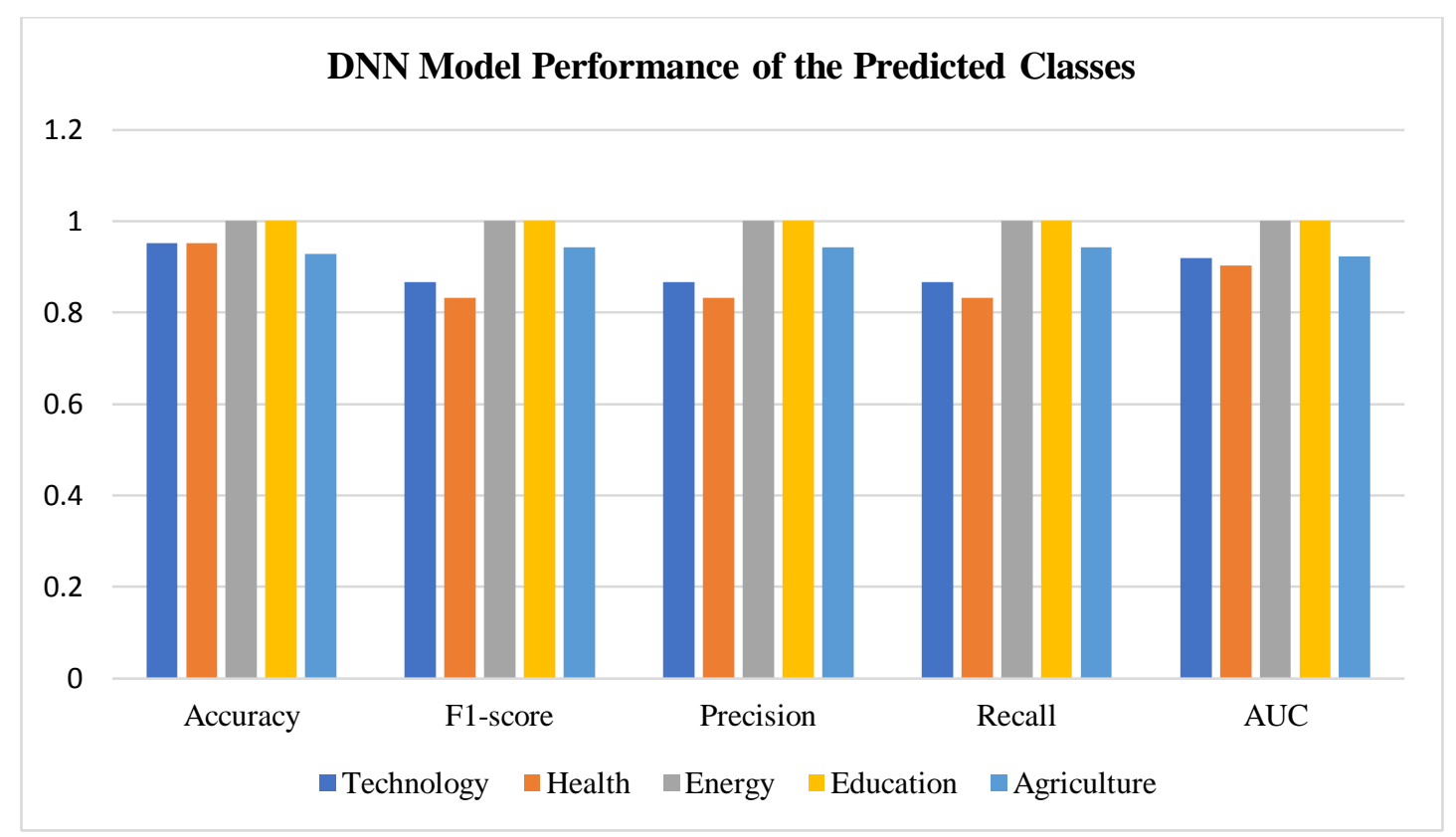

Fig 4: DNN Model Performance of the Five-Predicted Classes

\section{REFERENCES}

[1] E. T. Jackson and K. Harji, "Accelerating impact: Achievements, challenges and what's next in building the impact investing industry," Rockefeller Found., no. July, p. 86, 2012.

[2] A. K. Höchstädter and B. Scheck, "What's in a Name: An Analysis of Impact Investing Understandings by Academics and Practitioners," J. Bus. Ethics, vol. 132, no. 2, pp. 449-475, 2015.

[3] A. Bugg-levine and J. Emerson, "Impact Investing: Transforming How We Make Money while Making a Difference An," Innov. Technol. Gov. Glob., vol. 6, no. 3, pp. 9-18, 2011.

[4] J. Freireich and K. Fulton, Investing for social \& environmental impact. New York, NY:, 2009.

[5] GIIN, “Annual Impact Investor Survey 2019,” 2019.

[6] GIIN, “Annual Impact Investor Survey 2018,” 2018.

[7] A. Nicholls, "The social enterprise investment fund(SEIF) evaluation," no. December, 2010.

[8] M. Mendell and E. Barbosa, "Impact investing: a preliminary analysis of emergent primary and secondary exchange platforms," J. Sustain. Financ. Invest., vol. 3, no. 2, pp. 111-123, 2013.

[9] N. Reeder and A. Colantonio, "Measuring Impact and Non-financial Returns in Impact Investing: A Critical Overview of Concepts and Practice," EIBURS Work. Pap., no. 2013/01, pp. 1-44, 2013.

[10] L. Hehenberger, A.-M. Harling, and P. Scholten, A Practical Guide To Measuring and Managing Impact European Venture Philanthropy Association, no. June. 2015.

[11] S. Olsen and B. Galimidi, "Catalog of Approaches To Impact Measurement," Rockefeller Found., no. May, 2008.
[12] U. Grabenwarter and H. Liechtenstein, In Search of Gamma - An Unconventional Perspective on Impact Investing. 2012.

[13] Y. Saltuk, A. Bouri, A. Mudaliar, and M. Pease, "Perspectives on Progress," JP Morgan, no. January, pp. 1-28, 2013.

[14] I. So and A. Staskevicius, "Measuring the 'impact' in impact investing," no. August, pp. 1-31, 2015.

[15] L. Aquino- and S. Doran, "Impact investing: challenges of impact measuring," no. May, pp. 41-43, 2017.

[16] N. B. Verrinder, K. Zwane, D. Nixon, and S. Vaca, "Evaluative tools in impact investing: Three case studies on the use of theories of change," African Eval. J., vol. 6, no. 2, pp. 1-9, 2018.

[17] Tideline, "NAVIGATING IMPACT INVESTING," no. July, pp. 1-36, 2016.

[18] CISL, "In Search of Impact: Measuring the full value of capital," UK, 2016.

[19] GIIN, “Annual impact investor survey 2016.," New York, 2016.

[20] N. Watts and I. R. Scales, "Social impact investing, agriculture, and the financialization of development: Insights from sub-Saharan Africa," World Dev., vol. 130, p. $104918,2020$.

[21] R. Vargas, A. Mosavi, and R. Ruiz, "Deep Learning: A Review," Adv. Intell. Syst. Comput., no. October, 2018.

[22] F. Emmert-Streib, Z. Yang, H. Feng, S. Tripathi, and M. Dehmer, "An Introductory Review of Deep Learning for Prediction Models With Big Data," Front. Artif. Intell., vol. 3, no. February, pp. 1-23, 2020.

[23] A. Shrestha and A. Mahmood, "Review of deep learning algorithms and architectures," IEEE Access, vol. 7, pp. 53040-53065, 2019.

[24] K. Sirinukunwattana, S. E. A. Raza, Y. W. Tsang, D. R. 
J. Snead, I. A. Cree, and N. M. Rajpoot, "Locality Sensitive Deep Learning for Detection and Classification of Nuclei in Routine Colon Cancer Histology Images," IEEE Trans. Med. Imaging, vol. 35, no. 5, pp. 11961206, 2016.

[25] Y. LeCun, Y. Bengio, and G. Hinton, "Deep learning," Nature, vol. 521, no. 7553, p. 36, 2015.

[26] X. Z. Wang, T. Zhang, and R. Wang, "Noniterative deep learning: Incorporating restricted boltzmann machine into multilayer random weight neural networks," IEEE Trans. Syst. Man, Cybern. Syst., vol. 49, no. 7, pp. 12991308, 2019.

[27] Y. Bengio, "Deep learning of representations: Looking forward," in Proceedings of the 1st International Conference on Statistical Language and Speech Processing, 2013, pp. 1-37.

[28] Y. Bengio, A. Courville, and P. Vincent, "Representation learning: A review and new perspectives," IEEE Trans. Pattern Anal. Mach. Intell., vol. 35, no. 8, pp. 17981828, 2013.

[29] Y. Bengio and Y. Lecun, "Scaling Learning Algorithms towards AI," in Large Scale Kernel Machines, Eds., no. 34, L. Bottou, O. Chapelle, D. DeCoste, and J. Weston, Eds. Cambridge, MA: MIT Press, 2007, pp. 321-360.

[30] S. Ellis, T. Siesfeld, and D. Buelow, "Social capital : Measuring the community impact of corporate spending," Deloitte Insights, no. 24, 2019.

[31] R. A. Alenius, "Measurement Process in Impact Investing: State of Practice in Europe," Harvard Ext.
Sch., 2016.

[32] J. Miao and L. Niu, "A Survey on Feature Selection," Procedia Comput. Sci., vol. 91, no. Itqm, pp. 919-926, 2016.

[33] L. Breiman, "Random forests," Mach. Learn., vol. 45, no. 1, pp. 5-32, 2001.

[34] Q. Chen, Z. Meng, X. Liu, Q. Jin, and R. Su, "Decision variants for the automatic determination of optimal feature subset in RF-RFE," Genes (Basel)., vol. 9, no. 6, 2018.

[35] P. M. Granitto, C. Furlanello, F. Biasioli, and F. Gasperi, "Recursive feature elimination with random forest for PTR-MS analysis of agroindustrial products," Chemom. Intell. Lab. Syst., vol. 83, no. 2, pp. 83-90, 2006.

[36] N. K. Poona, A. Van Niekerk, R. L. Nadel, and R. Ismail, "Random Forest (RF) Wrappers for Waveband Selection and Classification of Hyperspectral Data," Appl. Spectrosc., vol. 70, no. 2, pp. 322-333, 2016.

[37] H. Jeon and S. Oh, "Hybrid-Recursive Feature Elimination for E ffi cient Feature Selection," Appl. Sci. pp. 1-8, 2020.

[38] D. West, "Neural network credit scoring models," Comput. Oper. Res., vol. 27, no. 11-12, pp. 1131-1152, 2000.

[39] P. Misra and A. S. Yadav, "Improving the classification accuracy using recursive feature elimination with crossvalidation," Int. J. Emerg. Technol., vol. 11, no. 3, pp. 659-665, 2020. 